\title{
Efficient Digital Signature Scheme for Internet of Things
}

\author{
${ }^{1}$ V. Muthukumaran, ${ }^{2}$ R. Murugesan, ${ }^{3}$ Lidia Victoria Segura Peña, ${ }^{4}$ Patricia María Zelaya Ycaza, \\ ${ }^{5}$ Gerber F. Incacari Sancho, ${ }^{6}$ Darci Gutiérrez Pinto \\ ${ }^{12}$ Department of Mathematics. School of Applied Sciences. REVA University, Bangalore-560064 \\ ${ }^{3}$ Universidad Tecnológica del Perú, Lima, Perú \\ ${ }^{4}$ Universidad Nacional Mayor de San Marcos, Lima, Perú \\ ${ }^{5}$ Universidad Nacionaldel Callao, Lima, Perú \\ ${ }^{6}$ Universidad AlasPeruanas, Arequipa, Perú. \\ ${ }^{1}$ muthu.v2404@gmail.com, ${ }^{2}$ contactmurugu@gmail.com, ${ }^{3} \mathrm{C} 19365 @$ utp.edu.pe, ${ }^{4}$ pzelaya @ unmsm.edu.pe, \\ ${ }^{5}$ gfincacaris@unac.edu.pe, ${ }^{6} \mathrm{~d} \_$gutierrez@doc.uap.edu.pe
}

Article History: Received: 11 January 2021; Accepted: 27 February 2021; Published online: 5 April 2021

\begin{abstract}
Apparently enthusiasm for the Internet of things (IoT) has as of late arrived at its top, with a lot of center from both the private and public parts. IoT, an innovation that empowers the trading of information through linkage among all items encompassing the client, can make new administrations. Information correspondence among objects isn't restricted to individual data, yet can likewise convey diverse information types, for example, detecting data gathered from the general condition. At the point when such information is gathered and utilized malevolently by an assailant, it is more defenseless against dangers than in traditional organization situations. In this article we proposed for digital signature scheme for Internet of Things.
\end{abstract}

Keywords: Internet of things (IoT), digital signature, data.

\section{Introduction}

The IoT are characterized as an organization all through information are gathered, prepared broke down the offer different types of assistance utilizing a progression of interconnected gadgets [1,]. The developing reception of IoT methods makes its application pervasive across different spaces, particularly with genuine applications. A portion of the significant uses of IoT framework incorporate brilliant homes, savvy urban areas, transportation, modern assembling, submerged asset the board, and medical services frameworks. The information created for example, distributed computing to re-appropriate capacity and calculation measures. That is the information gathered from IoT gadgets are put away over the distributed computing foundations for additional preparing and dynamic purposes. All in all, IoT gadgets utilize cloud-based framework (IaaS) administrations, as it doesn't just need information storerooms yet in addition need proficient information handling and calculation capabilities[3,4]. This makes the prerequisite of productive security components for secure administration of cloud based IoT frameworks.

Conveyed registering is an extraordinary perspective contribution a wide grouping of organizations over the web through a movement of unified preparing resources [5,6,11,12]. It engages one to store and access mystery data over the web instead of their local system plans. The NIST significance of passed on handling states that figuring is a prototypical for drawing in pervasive, good, on-request network authorization to an average pool of configurable enrolling assets that can be promptly provisioned and passed on with immaterial association exertion or ace network interaction[7,8,9,10]. By the day's end, the term conveyed registering used from wherever and at whatever point.

\section{Related Work}

The IoT grants to interface normal articles furnishing methods with perceiving, distinguishing, frameworks organization and taking care of capacities. Such limits grants objects with identifying and prompting capacities to talk with each other, and moreover with various machines and organizations around the Cyberspace, in order to accomplish tasks concerning IoT applications. Locales for new IoT applications fuse sharp homes, keen transportation systems, astute structures and shrewd condition noticing structure, among others. The IoT is prepared for precarious turn of events, with around 50 billion smart contraptions related with the Internet by 2020, and evaluated to make over $\$ 1.7$ trillion pay for consistently [1]. Progress of mechanized advances, for instance, ease while astoundingly capable sensors and processors, powerful distant shows, the adaptable turmoil and a swarm of new organizations and developed associations developing the fundamental application and the board programming.

IoT network is included a phenomenal amount of various contraptions advances, made withvarious merchants and for different purposes, similarly depicted by different limits. The toolsneednecessities to the extent taking care of capacity, memory, power smoothly, correspondence limit and UIs [2,3]. The use of obliged contraptions in networks routinely furthermore prompts necessities on the associations themselves. Regardless, there may similarly be impediments on networks that are by and large liberated from those of the centres. These necessities fuse high pack setback, minimalrealisticdata, nonappearance for forefront protection organizations and the 
astoundingly lopsided associations, including. Rule task to change associations to work in the standard establishment is a needed and crucial positive turn of events. Thus, in this one of a kind circumstance, imaginative work troubles are monstrous, and this emphatically applies to self-confidence.

\section{Proposed Method for Internet of Things}

Key Generation:

Apicks two arbitrary components $a, b \in N$ and a arbitrary $\vartheta(x) \in Z_{>0}[x]$ then $\vartheta(a)(\neq 0) \in N$ and then receipts $\vartheta(a)$ as her PK, calculate $y=\vartheta(a)^{r} b \vartheta(a)^{s}$ and publishes her public key $(a, b, y) \in N \times N \times N$.

Signature Generation:

A Completes of the next steps

Step 1

A picks the polynomial on $\vartheta(x) \in Z_{>0}[x]$ such that $\vartheta(a)(\neq 0) \in N$ and take $\vartheta(a)$ as salt.

Step 2

A calculate following steps

$\sigma=\vartheta(a)^{\wedge} \mathrm{r} b \vartheta(\mathrm{a})^{\wedge} \mathrm{s}$

$\psi=\vartheta(\mathrm{a})^{\wedge} \mathrm{r}[\mathrm{H}(\mathrm{M}) \sigma] \vartheta(\mathrm{a})^{\wedge} \mathrm{s}$

$\lambda=\vartheta(\mathrm{a})^{\wedge} \mathrm{r} \psi \vartheta(\mathrm{a})^{\wedge} \mathrm{s}$

$\rho=\vartheta(a)^{\wedge} \mathrm{r} \psi \delta(\mathrm{a})^{\wedge} \mathrm{s}$

$\alpha=\delta(\mathrm{a})^{\wedge} \mathrm{r} H(\mathrm{M}) \vartheta(\mathrm{a})^{\wedge} \mathrm{s}$

$\mathrm{U}=\vartheta(\mathrm{a})^{\wedge} \mathrm{r} \mathrm{H}(\mathrm{M}) \vartheta(\mathrm{a})^{\wedge} \mathrm{s}$

Then is the A signature on message and $\mathrm{B}$ verified.

Verification:

Validate the Alice's signature $(\sigma, \lambda, \rho, \alpha, U)$, Bdo the following

Step 1

To compute $V=\rho y^{-1} \alpha$.

Step 2

Bob accepts Alice's signature if $\sigma^{-1} U=\lambda^{-1} V$ then, he hand-me-down the signature.

\section{Security Analysis of IoT System}

Data forgery

Initially $\mathrm{E}$ substitutes the idea, with forgery one $M_{f}$. When signature which is attained by Bob $(\sigma, \lambda, \rho, \alpha, U)$. Expending data $M_{f}$ or $H\left(M_{f}\right)$, confirming the calculation

$$
(\sigma, \lambda, \rho, \alpha, U)
$$

is difficult, since or is totally complicated in the sign peers, but not in the confirmation procedure.

Then $\sigma^{-1} U=\lambda^{-1} V$ deprived of removing signature is not conceivable. Next effort to analyse the value $M_{f}$, for reasonable $H(M)$. But pertaining which is not conceivable due to assumption that occupation of hash is protected in graphically manner. So data is unacceptable that can't be designated with a signature that is not valid.

\section{Signature Repudiation:}

Considering the intend of Alice to recognition of refuses on his signature pertaining to some data which is valid $(\sigma, \lambda, \rho, \alpha, U)$ canister be counterfeit by $\mathrm{E}$ and she can sign the message $M$, with the signature that is forged $\left(\sigma_{f}, \lambda_{f}, \rho_{f}, \alpha_{f}, U_{f}\right)$ as a replacement. The confirmation technique as tails $V=\rho_{f} y^{-1} \alpha_{f}$

$V=\left[\vartheta(a)^{r} \psi \delta(a)^{s}\right]_{f}\left[\delta(a)^{-r} b^{-1} \delta(a)^{-s}\right]\left[\delta(a)^{r} H(M) \vartheta(a)^{s}\right]_{f}$.

Since $\left[\delta(a)^{r}\right]_{f} \cdot\left[\delta(a)^{r}\right] \neq I,\left[\delta(a)^{-s}\right]_{f} \cdot\left[\delta(a)^{-s}\right] \neq I$ where $\mathrm{I}$ is the individuality element in structure pertaining to the near-ring. Therefore $\left(\sigma^{-1} U\right)_{f} \neq\left(\lambda^{-1} V\right)$. Since the scheme for the signature ensures the property pertaining to repudiation. 


\section{Existential Forgery:}

Since $\mathrm{E}$ is analyzing to sign a message which is moved $M_{f}$. They must utilize the key by modifying with certain value $\left[\delta(a)^{r}\right]_{f}$. Consequently, she handles a issues with key considered to be public, as considering the NPSD which is retractable near ring. Also utilize every structure in schemes signature which are formed on non near ring and on basis of NPSD. Certain identification of these models are intractable as long as NPSD which is difficult in underlying structure of work. So structure new effective signatures, deprived of prior information of key which is considered to be private are impersistant. So as to Eve does not exist estimating signatures which are forgered.

\section{Result and Discussion}

The proposed algorithm is tested with different existing algorithms on the basis of encryption, decryption, authorization and test with respect to time cost, where the proposed algorithm has higher authorization time cost when compared with existing approaches. Some others systems as shown in graph has null authorization. The testing time cost for the proposed method has lower time cost.

\begin{tabular}{|c|c|c|c|c|c|c|c|}
\hline Schemes & Envi & $\mathrm{nm}$ & & & & & \\
\hline Yang et al & Con & Int & Aut & Non & $\begin{array}{l}\text { Pub } \\
\text { Ver }\end{array}$ & CipAut & PKI \\
\hline Karati et al & $\mathrm{NO}$ & NO & YES & $\mathrm{NO}$ & NO & YES & PKI \\
\hline Shen et al & NO & YE & NO & YES & NO & $\mathrm{NO}$ & $\mathrm{CLC}$ \\
\hline Ours & YES & $\begin{array}{l}\text { YE } \\
\text { S }\end{array}$ & YES & YES & YES & YES & Digital Signature \\
\hline
\end{tabular}

Fig.1 Comparison Security

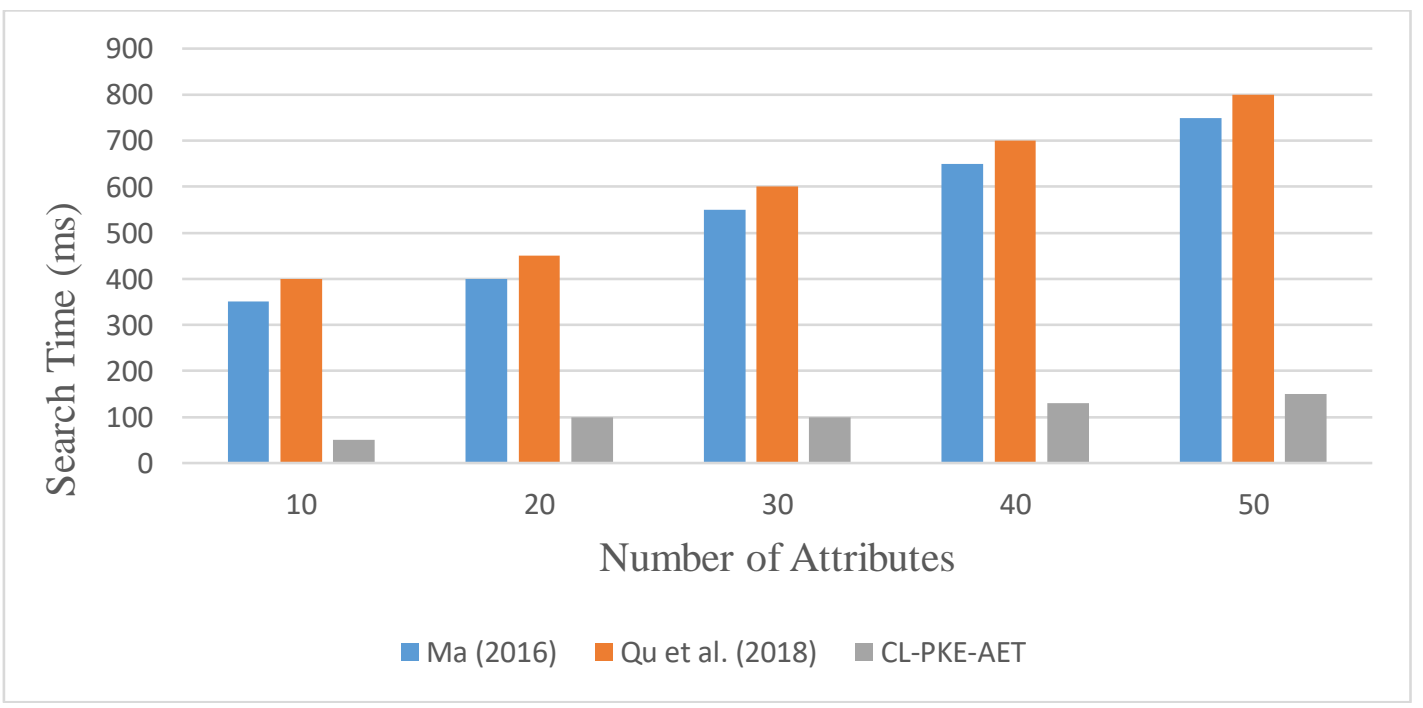

Fig.2 Time Complexity 


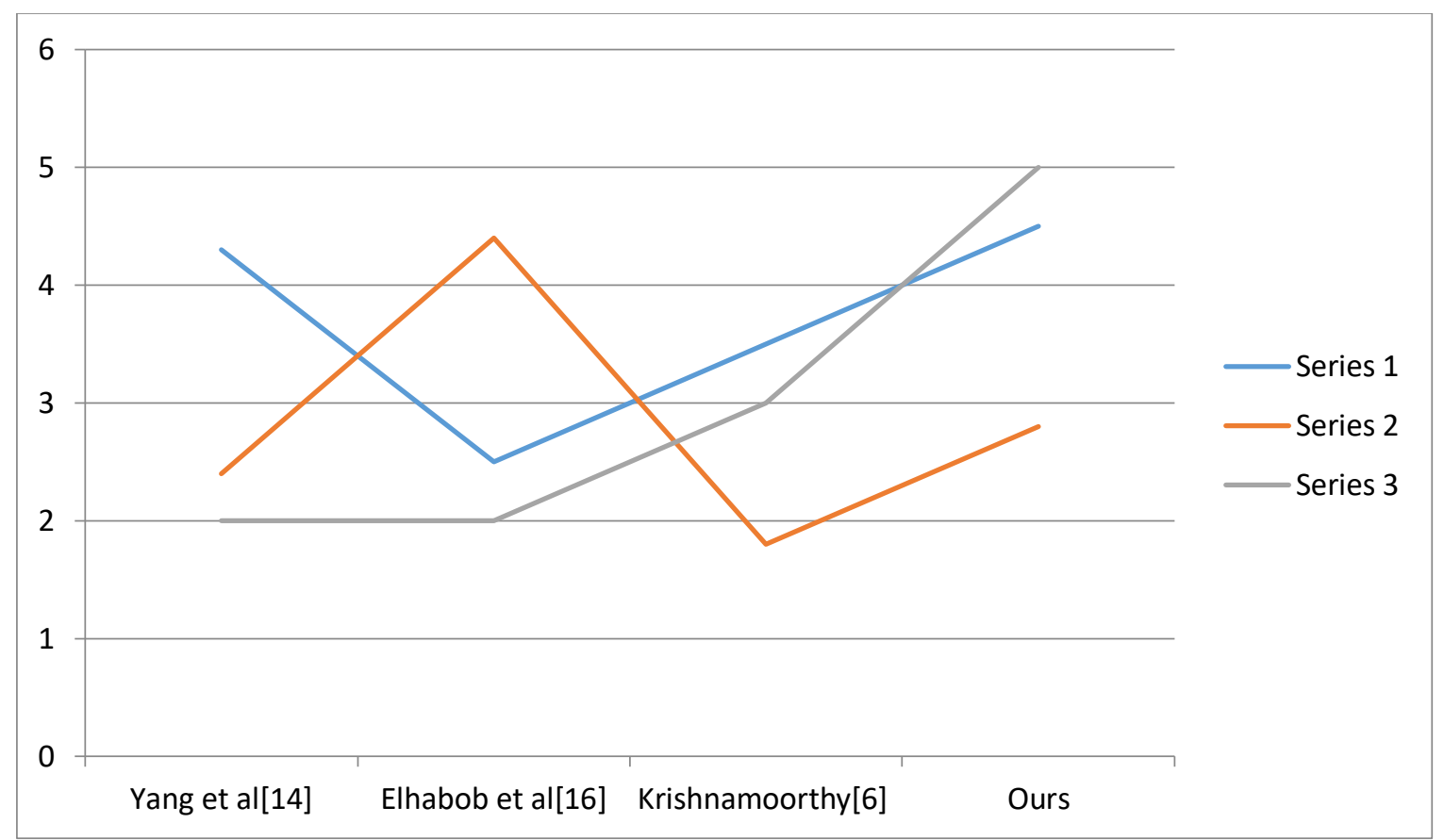

Fig.3 The comparison of computational complexity(time)

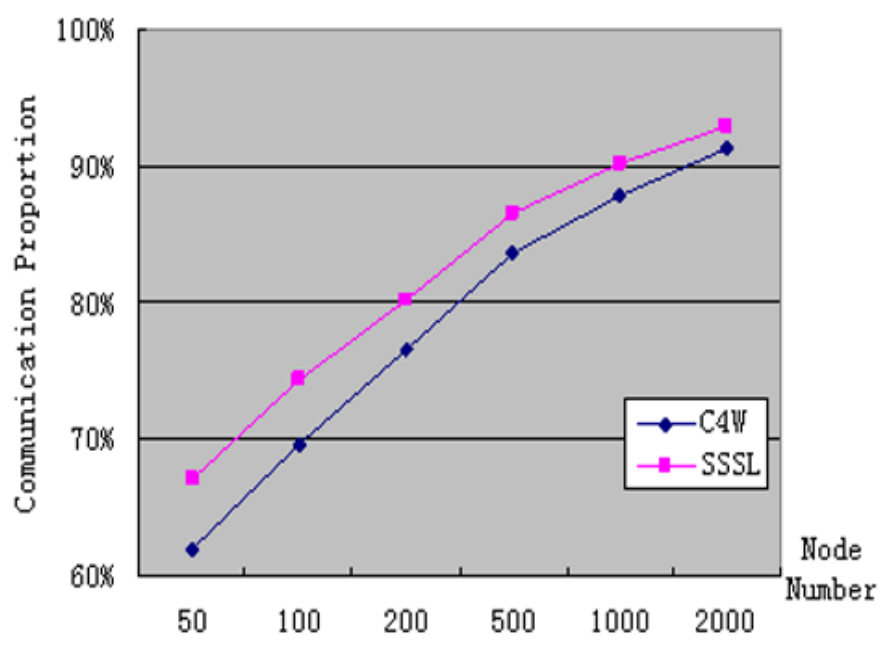

Fig.4 Communication Analysis

Security enhanced using the method is evaluted using different time metrics. Search time is one such metric which uses the attributes numbers based on the performance of the systems in providing security. Above figure describes the search time comparison made with different existing algorithms. Where our proposed security based method out performs other by minimizing the search time with increase in number of attributes.

\section{Conclusion}

In this article, we proposed computerized signature conspire dependent on near-ring. As far as anyone is concerned, this is the main mark conspire is particularly appropriate for IoT condition. The bogus rate for the proposed model is considerably less for distinguishing the malignant growth types. The over-fitting is decreased by acquiring right testing and preparing information for the model and utilizing PCA extraction method we further examined the element for development of execution. Also, this proposed model can be effortlessly utilized for the arrangement of multi-class dataset in various areas.

\section{References}

1. Yang, Y.; Wu, L.; Yin, G.; Li, L.; Zhao, H. A survey on security and privacy issues in Internet-ofThings. IEEE Internet Things J. 2017, 4, 1250-1258.

2. Shen, L.; Ma, J.; Liu, X.; Wei, F.; Miao, M. A secure and efficient id-based aggregate signature scheme 
${ }^{1}$ V. Muthukumaran, ${ }^{2}$ R. Murugesan, ${ }^{3}$ Lidia Victoria Segura Peña, ${ }^{4}$ Patricia María Zelaya Ycaza, ${ }^{5}$ Gerber F. Incacari Sancho, ${ }^{6}$ Darci Gutiérrez Pinto

for wireless sensor networks. IEEE Internet Things J. 2017, 4, 546-554.

3. Karati, A.; Islam, S.H.; Karuppiah, M. Provably secure and lightweight certificateless signature scheme for IIoT environments. IEEE Trans. Ind. Inform. 2018, 14, 3701-3711.

4. Yeh, K.H.; Su, C.; Choo, K.K.R.; Chiu, W. A novel certificateless signature scheme for smart objects in the Internet-of-Things. Sensors 2017, 17, 1001.

5. Huang, Y.; Zhang, X.; Yu, B. Efficient anti-replay identity-based signature scheme for wireless body area network. J. Cryptol. Res. 2017, 4, 447-457.

6. S. KRISHNAMOORTHY, V. MUTHUKUMARAN, J. YU, B. BALAMURUGAN: A Secure Privacy Preserving Proxy re-encryption Scheme for IoT Security using Near-ring, In Proceedings of the 2019 the International Conference on Pattern Recognition and Artificial Intelligence,ACM, (2019), 27-32.

7. V. MUTHUKUMARAN, D. EZHILMARAN: Authenticated Group Key Agreement Protocol Based on Twisted Conjugacy Root Extraction Problem in Near-Ring, Journal of Computationaland Theoretical Nanoscience., 15(6-7) (2018), 2023-2026.

8. V. MUTHUKUMARAN, D. EZHILMARAN, G. S. G. N. ANJANEYULU: Efficient Authentication Scheme Based on the Twisted Near-Ring Root Extraction Problem, Advances in Algebraand Analysis, 5 (2018), 37-42.

9. D. EZHILMARAN, V. MUTHUKUMARAN: Key Exchange Protocol Using Decomposition Problem In Near-Ring, Advances in Algebra and Analysis, 29(1) (2016), 123-127.

10. D. Ezhilmaran, V. Muthukumaran: Authenticated group key agreement protocol based on twist conjugacy problem in near-rings, Wuhan University Journal of Natural Sciences, 22(6) (2017), 472-476.

11. V. Muthukumaran, D. Ezhilmaran: Efficient authentication scheme based on nearing root extraction problem, Materials Science and Engineering Conference Series, 15(2017), 042137.

12. V. MUTHUKUMARAN, D. EZHILMARAN, I. MUCHTADI-ALAMSYAH, R. UDHAYAKUMAR, A. MANICKAM: New public key cryptosystem based on combination of NREP and CSPin noncommutative near-ring, Journal of Xi'an University of Architecture and Technology, 12(3) (2020), 4534-4539.

13. V. Muthukumaran, D. Ezhilmaran and M. Adhiyaman A SECURE AND ENHANCED PUBLIC KEY CRYPTOSYSTEM USING DOUBLE CONJUGACY SEARCH PROBLEM NEAR-RING, Advances in Mathematics: Scientific Journal , 9(3), 1389-1395, 2020.

14. Yang, X.; Chen, C.; Ma, T.; Li, Y.; Wang, C. An improved certificateless aggregate signature scheme for vehicular ad-hoc networks. In Proceedings of the IEEE 3rd Advanced Information Technology, Electronic and Automation Control Conference, Chongqing, China, 12-14 October 2018; pp. 23342338.

15. Yang, X.D.; Xiao, L.K.; Chen, C.L.; Wang, C.F. A strong designated verifier proxy re-signature scheme for IoT environments. Symmetry 2018, 10, 580.

16. Elhabob, R., Zhao, Y., Sella, I. and Xiong, H., 2020. An efficient certificateless public key cryptography with authorized equality test in IIoT. Journal of Ambient Intelligence and Humanized Computing, 11(3), pp.1065-1083. 